\title{
Clarifying the link between ibuprofen and hypogonadism
}

\author{
Bernard Jégou and David M. Kristensen
}

In a 2018 study in the Proceedings of the National Academy of Sciences of the USA ${ }^{1}$, we concluded that ibuprofen alters pituitarytestis endocrine balance via selective transcriptional repression in the testis, resulting in compensated hypogonadism. This paper was discussed in a commentary by Nangia and Jensen (Ibuprofen and hypogonadism bench to bedside to misinterpreted hype? Nat. Rev. Urol. 15, 268-269 (2018)) $)^{2}$. Our study involved a clinical trial performed in men aged 18-35 years and exposure of adult human testes explants and a human steroidogenic cell line to ibuprofen, which is frequently used as an analgesic and often used prophylactically at high doses by athletes ${ }^{3,4}$.

In their commentary, Nangia and Jensen $^{2}$ claim that the original paper argues that the class of medication to which ibuprofen belongs is the specific cause of the reduction of overall male reproductive health and male reproductive deterioration. However, this statement is contradicted by the actual content of the paper, which emphasizes that establishing the possible effect of ibuprofen on sperm production and fertility would require the design of specific experiments. Contextualization of our findings by mentioning the general concern about declining male reproductive health should not be confused with our speculations regarding the significance of our data.

The authors also asserted that our data were difficult to interpret because they were part of a larger study ${ }^{5}$. However, our data showed a consistent increase in plasma luteinizing hormone $(\mathrm{LH})$ in the ibuprofen-treated group ( $+23 \%$ and $P \leq 0.05$ after 14 days of ibuprofen; $+33 \%$ and $P \leq 0.01$ after 44 days of ibuprofen) compared with placebo ${ }^{1}$.

We are disappointed that Nangia and Jensen suggested that we "inadvertently provided fuel to the media fire", thereby contributing to "misinterpreted hype". A press release was issued by the original journal and media interest was likely fuelled by the widespread use of ibuprofen and analgesics in general. Although we requested access to media content before publication to verify their accuracy, the headlines were sometimes flashy clickbait, designed to drive traffic as is often the case in such circumstances. Other media representatives, who did not attempt to contact us, made false statements, and our study was sometimes distorted by the internet and social media.

Mild analgesics have an important medical role. However, the time has come to accept that their widespread and increasing use is not without consequences, some of which have already been identified, for example, for ibuprofen use during pregnancy or in adulthood ${ }^{3,6}$. Whether ibuprofen can have direct effects on the sperm count in young men still needs to be determined, and whether ibuprofen or other analgesics might also induce a shift from compensated hypogonadism to primary hypogonadism in ageing men ${ }^{7}$ requires particular vigilance.

Bernard Jégou ${ }^{*}$ and David M. Kristensen ${ }^{1,2}$ 'Univ Rennes, Inserm, EHESP, Irset (Institut de recherche en santé, environnement et travail) - UMR_ S1085, F-35000 Rennes, France.

${ }^{2}$ Department of Neurology, Danish Headache Center, Rigshospitalet, University of Copenhagen, Copenhagen, Denmark. *e-mail: bernard.jegou@inserm.fr https://doi.org/10.1038/s41585-019-0146-x

1. Kristensen, D. M. et al. Ibuprofen alters human testicular physiology to produce a state of compensated hypogonadism. Proc. Natl Acad. Sci. USA 115, E715-E724 (2018)

2. Nangia, A. K. \& Jensen, D. Ibuprofen and hypogonadism - bench to bedside to misinterpreted hype? Nat. Rev. Urol. 15, 268-269 (2018).

3. Küster, M. et al. Consumption of analgesics before a marathon and the incidence of cardiovascular, gastrointestinal and renal problems: a cohort study. BMJ Open 3, e002090 (2013).

4. Manoukian, M. A. C. et al. Topical administration of ibuprofen for injured athletes: considerations, formulations, and comparison to oral delivery. Sports Med. Open 3, 36 (2017).

5. Mackey, A. L. et al. Activation of satellite cells and the regeneration of human skeletal muscle are expedited by ingestion of nonsteroidal anti-inflammatory medication. FASEB J. 30, 2266-2281 (2016).

6. Kristensen, D. M. et al. Analgesic use prevalence biomonitoring and endocrine and reproductive effects. Nat. Rev. Endocrinol. 12, 381-393 (2016).

7. Tajar, A et al. Characteristics of androgen deficiency in late-onset hypogonadism: results from the European Male Aging Study (EMAS). J. Clin. Endocrinol. Metab. 97, 1508-1516 (2012).

Competing interests

The authors declare no competing interests. 